\title{
Semi-Infinite Optimization: Structure and Stability of the Feasible Set ${ }^{1}$
}

\author{
H. T. JoNGEN, ${ }^{2}$ F. TWILT, ${ }^{3}$ AND G. W. WEBER ${ }^{4}$ \\ Communicated by O. L. Mangasarian
}

\begin{abstract}
The problem of the minimization of a function $f: \mathbb{R}^{n} \rightarrow \mathbb{R}$ under finitely many equality constraints and perhaps infinitely many inequality constraints gives rise to a structural analysis of the feasible set $M[H, G]=\left\{x \in \mathbb{R}^{n} \mid H(x)=0, G(x, y) \geq 0, y \in Y\right\}$ with compact $Y \subset$ $\mathbb{R}^{r}$. An extension of the well-known Mangasarian-Fromovitz constraint qualification (EMFCQ) is introduced. The main result for compact $M[H, G]$ is the equivalence of the topological stability of the feasible set $M[H, G]$ and the validity of EMFCQ. As a byproduct, we obtain under EMFCQ that the feasible set admits local linearizations and also that $M[H, G]$ depends continuously on the pair $(H, G)$. Moreover, EMFCQ is shown to be satisfied generically.
\end{abstract}

Key Words. Semi-infinite optimization, topological stability, reduction principle, structure of the feasible set, genericity.

\section{Introduction}

An optimization problem of semi-infinite type has the following specific property: the feasible set is described by means of infinitely many inequality constraints. In this paper, we consider semi-infinite optimization problems of the following form:

(SIP) minimize $f$ on $M[H, G]$,

$$
\begin{aligned}
& M[H, G]=\left\{x \in \mathbb{R}^{n} \mid H(x)=0, G(x, y) \geq 0, \text { for all } y \in Y\right\}, \\
& Y \subset \mathbb{R}^{r} \text { compact. }
\end{aligned}
$$

\footnotetext{
${ }^{1}$ The authors would like to thank Rainer Hettich and Doug Ward for fruitful discussions. Moreover, the authors are indebted to the anonymous referees for their valuable comments. ${ }^{2}$ Professor, Aachen University of Technology, Department of Mathematics, Aachen, Germany. ${ }^{3}$ Senior Lecturer, University of Twente, Department of Applied Mathematics, Enschede, The Netherlands.

${ }^{4}$ Scientific Assistant, Aachen University of Technology, Department of Mathematics, Aachen, Germany.
} 
In (1), the mappings $f: \mathbb{R}^{n} \rightarrow \mathbb{R}, \quad H=\left(h_{1}, \ldots, h_{m}\right): \mathbb{R}^{n} \rightarrow \mathbb{R}^{m}, \quad m<n$, $G: \mathbb{R}^{n} \times \mathbb{R}^{r} \rightarrow \mathbb{R}$ are assumed to be continuously differentiable.

We are interested in the local structure of the feasible set $M[H, G]$ and its topological stability depending on the pair $(H, G)$. The ideas in this paper extend and generalize the work done in Ref. 1. In fact, in Ref. 1 the set $Y \subset \mathbb{R}^{r}$, the index set of inequality constraints, was assumed to be a finite set. In the present paper, however, the index set $Y$ might be a continuum, such as an interval, a rectangle, or more generally a compact manifold with boundary.

A central role will be played by an appropriate extension of the so-called Mangasarian-Fromovitz constraint qualification (cf. Ref. 2). Under assumption of the extended Mangasarian-Fromovitz constraint qualification (EMFCQ), it will be shown that the set $M[H, G]$ is a Lipschitzian manifold with boundary. Moreover, if $M[H, G]$ is compact, then for all $(\tilde{H}, \tilde{G})$ sufficiently close to $(H, G)$ (the topology to be specified below), the corresponding set $M[\tilde{H}, \tilde{G}]$ is homeomorphic with $M[H, G]$ if and only if EMFCQ is satisfied on $M[H, G]$. This characterization will be our main result. The compactness of $M[H, G]$ and the validity of EMFCQ also guarantee locally the continuity of the set-valued mapping $(H, G) \mapsto M[H, G]$. Finally, we prove that the fulfilment of EMFCQ turns out to be generically true.

A first and easy approach to the study of the structure of the feasible set $M[H, G]$ is that of a local reduction to a set described by means of a finite number of differentiable constraints. This can be done as follows (cf. Refs. 3 and 4). Let us introduce the marginal function,

$$
\phi_{G}(x)=\min _{y \in Y} G(x, y),
$$

and the extremal set $E_{G}(x)$ (the index set of active inequality constraints),

$$
E_{G}(x)=\{y \in Y \mid G(x, y)=0\}, \quad x \in M[H, G] .
$$

An easy but important observation is the following: for $\bar{x} \in M[H, G]$, each $y \in E_{G}(\bar{x})$ is a global minimum for $G(\bar{x}, \cdot)_{\mid Y}$. Now, if each $y \in E_{G}(\bar{x})$ is nondegenerate (to be specified below), then the set $E_{G}(\bar{x})$ is discrete, and hence finite (recall that $Y$ is a compact set), say $E_{G}(\bar{x})=\left\{\bar{y}^{1}, \ldots, \bar{y}^{p}\right\}$. Then, application of the implicit function theorem around each point $\left(\bar{x}, \bar{y}^{i}\right)$, gives rise to a local minimum $y^{i}(x)$ for $G(x,)_{\mid Y}$ depending on $x$. As a consequence, we obtain in a neighborhood $U$ of $\bar{x}$ the representation

$$
\phi_{G}(x)=\min _{i=1, \ldots, p} \phi_{G}^{i}(x), \quad \phi_{G}^{i}(x)=G\left(x, y^{i}(x)\right),
$$

and the set $M[H, G] \cap \mathscr{U}$ can be described by means of a finite number of 
differentiable constraints,

$$
M[H, G] \cap \mathscr{U}=\left\{x \in \mathscr{U} \mid H(x)=0, \phi_{G}^{i}(x) \geq 0, i=1, \ldots, p\right\} .
$$

Unfortunately this latter reduction principle cannot be applied at all feasible points. In fact, we will show, using ideas from singularity theory (cf. Ref. 5), that the violation of the assumptions for applying the reduction principle might be stable under perturbations of the defining pair $(H, G)$. In spite of the latter negative result, it was shown in Ref. 6 that the reduction principle is generically applicable in a neighborhood of the local minima of (SIP). In the present paper, we use the reduction principle at certain points in the proofs in order to be able to exploit the results from Ref. 1.

This paper is organized as follows. In Section 2, we state the main theorems and discuss the reduction principle. Section 3 contains several lemmas and the proof of the theorems.

\section{Definitions and Main Results}

Let $C^{k}\left(\mathbb{R}^{n}, \mathbb{R}^{m}\right)$ denote the space of $k$-times continuously differentiable mappings from $\mathbb{R}^{n}$ to $\mathbb{R}^{m}$.

The index set $Y$ in (1) will be described by means of a finite number of smooth (in)equality constraints,

$$
Y=\left\{y \in \mathbb{R}^{r} \mid U(y)=0, V(y) \geq 0\right\}, \quad Y \text { compact, }
$$

where

$$
\begin{aligned}
& U=\left(u_{1}, \ldots, u_{\alpha}\right) \in C^{\infty}\left(\mathbb{R}^{r}, \mathbb{R}^{\alpha}\right), \quad \alpha<r, \\
& V=\left(v_{1}, \ldots, v_{\beta}\right) \in C^{\infty}\left(\mathbb{R}^{r}, \mathbb{R}^{\beta}\right) .
\end{aligned}
$$

Throughout this paper, it is assumed that the linear independence constraint qualification ( $L I C Q$ ) is satisfied at all $y \in Y$, i.e.,

(LICQ) $\left\{D u_{i}(\bar{y}), D v_{j}(\tilde{y}) ; \dot{i} \in A, j \in B_{0}(\bar{y})\right\}$ is a linearly independent set, $\bar{y} \in Y$, where

$$
\begin{aligned}
& A=\{1, \ldots, \alpha\}, \quad B=\{1, \ldots, \beta\}, \\
& B_{0}(\bar{y})=\left\{j \in B \mid v_{j}(\bar{y})=0\right\},
\end{aligned}
$$

and where $D u_{i}(\bar{y})$ stands for the row vector of first partial derivatives of $u_{i}$ evaluated at the point $\bar{y}, D v_{j}(\bar{y})$ being defined similarly.

The validity of (LICQ) on $Y$ assures that the set $Y$ is a smooth manifold with boundary and corners (cf. Ref. 7 ), such as an interval, a rectangle, etc.

An extension of (LICQ) above can be formulated (and will be used) for sets of the type $M[H, G]$ as introduced in Section 1 ( $D_{x}$ denoting partial differentiation with respect to $x$ ). 
Definition 2.1. The extended linear independence constraint qualification (ELICQ) is said to hold at $\bar{x} \in M[H, G]$ if the conditions below are satisfied:

(ELICQ1) the extremal set $E_{G}(\bar{x})$ is finite;

(ELICQ2) the set $\left\{D h_{i}(\bar{x}), D_{x} G(\bar{x}, y) ; i=1, \ldots, m, y \in E_{G}(\bar{x})\right\}$ is linearly independent.

Note that, formally, condition (ELICQ2) implies (ELICQ1). The following constraint qualification (EMFCQ) will play a crucial role in this paper; it is not difficult to see that (ELICQ) implies the validity of (EMFCQ), the converse being false.

Definition 2.2. The extended Mangasarian-Fromovitz constraint qualification (EMFCQ) is said to hold at $\bar{x} \in M[H, G]$ if the conditions below are satisfied:

(EMFCQ1) rank $D H(\bar{x})=m$;

(EMFCQ2) there exists a vector $\xi \in \mathbb{R}^{n}$ satisfying

$$
\begin{aligned}
& D H(\bar{x}) \cdot \xi=0, \\
& D_{x} G(\bar{x}, y) \cdot \xi>0, \quad \text { for all } y \in E_{G}(\bar{x}) .
\end{aligned}
$$

A vector $\xi \in \mathbb{R}^{n}$ satisfying ( $8 \mathrm{a}$ ) and ( $8 \mathrm{~b}$ ) will be called an EMF-vector at $\tilde{x}$.

Before stating our first theorem, we have to introduce the notion of a Lipschitzian manifold, which is a special type of topological manifold. Let $\mathbb{H}^{n}$ denote the nonnegative orthant in $\mathbb{R}^{n}$, i.e.,

$$
\mathbb{H}^{n}=\left\{x \in \mathbb{R}^{n} \mid x \geq 0\right\} \text {. }
$$

Definition 2.3. A subset $M \subset \mathbb{R}^{n}$ is called a Lipschitzian manifold (with boundary) of dimension $k$ if, for each $\vec{x} \in M$, there exist open neighborhoods $\mathscr{U}$ of $\bar{x}$ and $\mathscr{V}$ of the origin and a bijective mapping $\Phi: \mathscr{U} \rightarrow \mathscr{V}$, with both $\Phi$ and $\Phi^{-1}$ Lipschitz-continuous, sending $\bar{x}$ onto the origin, such that either

$$
\Phi(\mathscr{U} \cap M)=\mathscr{V} \cap\left(\left\{O_{n-k}\right\} \times \mathbb{R}^{k}\right)
$$

or

$$
\Phi(\mathscr{U} \cap M)=\mathscr{V} \cap\left(\left\{O_{n-k}\right\} \times \mathbb{H}^{1} \times \mathbb{R}^{k-1}\right) ;
$$

in the latter case, $\bar{x}$ is called boundary point. The set of all boundary points will be denoted by $\partial M$. 
Theorem 2.1. Manifold Theorem. Suppose that (EMFCQ) is satisfied at all points of $M[H, G]$. Then, $M[H, G]$ is a Lipschitzian manifold (with boundary) of dimension $n-m$, and the boundary $\partial M[H, G]$ equals $\left\{x \in \mathbb{R}^{n} \mid H(x)=0, \phi_{G}(x)=0\right\}$.

In the following theorems, we use several topologies which we now introduce. For $r \leq k$, the space $C^{k}\left(\mathbb{R}^{n}, \mathbb{R}\right)$ will be topologized by means of the strong $C^{r}$-topology (also called $C^{r}$ - Whitney topology), denoted by $C_{s}^{r}$ (cf. Ref. 8). In fact, for finite $r$, the $C_{s}^{r}$-topology is generated by allowing perturbations of the functions and their derivatives up to order $r$ which are controlled by continuous positive functions $\in(\cdot): \mathbb{R}^{n} \rightarrow \mathbb{R}$ (rather than positive constants $\epsilon$ ); note that the infimum of $\epsilon(\cdot)$ over $\mathbb{R}^{n}$ might be zero. The $C_{s}^{\infty}$-topology for $C^{\infty}\left(\mathbb{R}^{n}, \mathbb{R}\right)$ is generated by means of the union of the bases for the $C_{s}^{r}$-topology, $r=0,1,2, \ldots$ The $C_{s}^{r}$-topology for $C^{k}\left(\mathbb{R}^{n}, \mathbb{R}^{m}\right)$ is obtained by means of the product-topology induced by $C_{s}^{r}$ on the $m$-fold product $C^{k}\left(\mathbb{R}^{n}, \mathbb{R}\right) \times \cdots \times C^{k}\left(\mathbb{R}^{n}, \mathbb{R}\right)$.

In order to state our continuity theorem, we need the notions of lower and upper semicontinuity of a point-to-set mapping $\mathcal{M}$ from a topological space $T$ into the family $\mathscr{P}\left(\mathbb{R}^{n}\right)$ of all subsets of $\mathbb{R}^{n}$. Following Berge, Ref. 9, we call $\mathscr{M}$ lower semicontinuous (1.s.c.) at $\bar{v} \in T$ if, for any open set $\mathscr{U} \subset \mathbb{R}^{n}$ with $\mathscr{M}(\bar{v}) \cap \mathcal{U} \neq \varnothing$, there exists a neighborhood $\mathscr{V}$ of $\bar{v}$ such that $\mathscr{M}(v) \cap \mathcal{U} \neq \varnothing$ whenever $v \in \mathscr{V}$. The mapping $\mathcal{M}$ is said to be upper semicontinuous (u.s.c.) at $\bar{v} \in T$ if, for any open set $\mathscr{U} \subset \mathbb{R}^{n}$ with $M(\bar{v}) \subset \mathcal{U}$, there exists a neighborhood $\mathscr{V}$ of $\bar{v}$ such that $M(v) \subset \mathscr{U}$ whenever $v \in \mathscr{V}$.

For an extensive study of these two notions (and of related ones) in parametric optimization, see Ref. 10.

The family of all compact subsets of $\mathbb{R}^{n}$ endowed with the Hausdorff metric (cf. Ref. 9) is denoted by $\mathscr{P}_{c}\left(\mathbb{R}^{n}\right)$.

Now, we have the following theorem.

Theorem 2.2. Continuity Theorem. Let $H \in C^{2}\left(\mathbb{R}^{n}, \mathbb{R}^{m}\right)$, and suppose that (EMFCQ) is satisfied for all $x \in M[H, G]$. Then, there exists a $C_{s}^{1}$-neighborhood $\mathscr{O} \subset C^{2}\left(\mathbb{R}^{n}, \mathbb{R}^{m}\right) \times C^{1}\left(\mathbb{R}^{n} \times \mathbb{R}^{r}, \mathbb{R}\right)$ such that the set-valued mapping $\mathscr{M}$,

$$
\mathscr{M}:(\tilde{H}, \tilde{G}) \mapsto M[\tilde{H}, \tilde{G}],
$$

is both upper semicontinuous and lower semicontinuous at all $(\tilde{H}, \tilde{G}) \in \mathcal{O}$. Moreover, if in addition $M[H, G]$ is compact, then $O$ can be chosen in such a way that $\mathscr{M}$ maps $\mathscr{O}$ to $\mathscr{P}_{c}\left(\mathbb{R}^{n}\right)$ and is continuous as well.

We emphasize that continuity of a mapping $F: T \rightarrow \mathscr{P}_{\mathrm{c}}\left(\mathbb{R}^{n}\right)$, with $T$ a topological space, cannot prevent bifurcations of the set $F(t)$, when $t$ traverses $T$. This is illustrated in the next example. 
Example 2.1. Let $T=(-\infty, 1)$, and define $F: T \rightarrow \mathscr{P}_{c}\left(\mathbb{R}^{n}\right)$ as follows:

$$
F(t)=\left\{x \in[-1,1] \mid x^{2}-t \geq 0\right\} .
$$

The continuity of $F$ is easily verified. Note that, when $t$ traverses zero from negative to positive values, the set $F(t)$ bifurcates from one connected component into two components.

The subsequent stability theorem, however, shows that, under the assumptions of Theorem 2.2, bifurcations of the feasible set do not occur.

Definition 2.4. Let $H \in C^{2}\left(\mathbb{R}^{n}, \mathbb{R}^{m}\right)$. The set $M[H, G]$ is called stable if there exists a $C_{s}^{1}$-neighborhood $O$ of $(H, G)$ in $C^{2}\left(\mathbb{R}^{n}, \mathbb{R}^{m}\right) \times$ $C^{l}\left(\mathbb{R}^{n} \times \mathbb{R}^{r}, \mathbb{R}\right)$ such that, for every $(\tilde{H}, \tilde{G}) \in \mathcal{O}$, the corresponding feasible set $M[\tilde{H}, \tilde{G}]$ is homeomorphic with $M[H, G]$.

Now, we can state our main theorem.

Theorem 2.3. Stability Theorem. Let $H \in C^{2}\left(\mathbb{R}^{n}, \mathbb{R}^{m}\right)$, and suppose that $M[H, G]$ is compact. Then, the feasible set $M[H, G]$ is stable if and only if (EMFCQ) holds at every point $x \in M[H, G]$.

A subset of a topological space $T$ is called generic if it contains a countable intersection of open and dense subsets. If $T$ is a so-called Baire space, then a generic subset is also dense. In particular, the space $C^{k}\left(\mathbb{R}^{n}, \mathbb{R}\right)$ endowed with the $C_{s}^{k}$-topology is a Baire space (cf. Refs. 8 and 11).

Theorem 2.4. Genericity Theorem.

(a) Let $C^{\infty}\left(\mathbb{R}^{n}, \mathbb{R}^{m}\right) \times C^{\infty}\left(\mathbb{R}^{n} \times \mathbb{R}^{r}, \mathbb{R}\right)$ be endowed with the $C_{s}^{\infty}$-topology. Then, there exists a generic subset $\mathscr{A} \subset$ $C^{\infty}\left(\mathbb{R}^{n}, \mathbb{R}^{m}\right) \times C^{\infty}\left(\mathbb{R}^{n} \times \mathbb{R}^{r}, \mathbb{R}\right)$ such that, for each $(H, G) \in \mathscr{A}$, it holds that (ELICQ) is fulfilled at every $x \in M[H, G]$.

(b) Let $\mathscr{F}$ be the subset of $C^{1}\left(\mathbb{R}^{n}, \mathbb{R}^{m}\right) \times C^{1}\left(\mathbb{R}^{n} \times \mathbb{R}^{r}, \mathbb{R}\right)$ consisting of those pairs $(H, G)$ for which (EMFCQ) holds at all points $x \in$ $M[H, G]$. Then, $\mathscr{F}$ is $C_{s}^{1}$-open and $C_{s}^{1}$-dense.

We shall end this section with a discussion on the reduction principle. For omitted details, we refer to Refs. 4,11 , and 12 . For our aim, we now suppose that $G \in C^{2}\left(\mathbb{R}^{n} \times \mathbb{R}^{r}, \mathbb{R}\right)$ and recall that, for $\bar{x} \in M[H, G]$, each point $\bar{y} \in E_{G}(\bar{x})$ is a global minimum for $G(\bar{x}, \cdot)_{\mid Y}$.

Definition 2.5. A point $\bar{y} \in Y$ is called a critical point for $G(\bar{x}, \cdot)_{\mid Y}$ if there exist reals $\bar{\lambda}_{i}, i \in A$, and $\bar{\mu}_{j}, j \in B_{0}(\bar{y})$, such that

$$
D_{y} G(\bar{x}, \bar{y})=\sum_{i \in A} \bar{\lambda}_{i} D u_{i}(\tilde{y})+\sum_{j \in B_{0}(\bar{y})} \bar{\mu}_{j} D v_{j}(\bar{y}) \text {. }
$$


A critical point $\bar{y}$ for $G\left(\bar{x}_{3}\right)_{\mid \gamma}$ is said to be nondegenerate the conditions below hold:

(ND1) $\bar{\mu}_{j} \neq 0$ for all $j \in B_{0}(\bar{y})$;

(ND2) the matrix $V^{T} \cdot D_{y}^{2} L(\bar{x}, \bar{y}) \cdot V$ is nonsingular, where

$$
L(x, y)=G(x, y)-\sum_{i \in A} \bar{\lambda}_{i} u_{i}(y)-\sum_{j \in B_{0}(\bar{y})} \bar{\mu}_{j} v_{j}(y)
$$

and where the columns of the matrix $V$ constitute a basis for the tangent space $T(\bar{y})$,

$$
T(\bar{y})=\bigcap_{i \in A} \operatorname{Ker} D u_{i}(\tilde{y}) \cap \bigcap_{j \in B_{0}(\bar{y})} \operatorname{Ker} D v_{j}(\bar{y})
$$

In particular, a point $\bar{y} \in Y$ is a nondegenerate local minimum for $G\left(\vec{x}_{3} \cdot\right) \mid Y$ if, besides (12), (ND1), and (ND2), the numbers $\bar{\mu}_{j}$ are positive and moreover $V^{T} \cdot D_{y}^{2} L(\bar{x}, \bar{y}) \cdot V$ is positive definite.

In the above definition, $D_{y}^{2} L$ stands for the matrix of second-order partial derivatives of $L$ with respect to $y$, and

$$
\operatorname{Ker} D u_{i}(\bar{y})=\left\{\eta \in \mathbb{R}^{r} \mid D u_{i}(\bar{y}) \eta=0\right\} \text {. }
$$

Suppose that $\bar{y} \in Y$ is a nondegenerate critical point for $G(\bar{x}, *)_{Y}$. Let us introduce the critical point map $\mathscr{T}$,

$$
\mathscr{T}:\left[\begin{array}{c}
y \\
\lambda \\
\mu \\
x
\end{array}\right] \mapsto\left[\begin{array}{cl}
D_{y}^{\mathrm{T}} G(x, y)-\sum_{i \in A} \lambda_{i} D^{\mathrm{T}} u_{i}(y)-\sum_{j \in B_{0}(y)} \mu_{j} D^{\mathrm{T}} v_{j}(y) \\
-u_{i}(y), & i \in A \\
-v_{j}(y), & j \in B_{0}(\bar{y})
\end{array}\right]
$$

where $\lambda$ and $\mu$ are $|A|$-vectors and $\left|B_{0}(\bar{y})\right|$-vectors, respectively. We note that $\mathscr{T}$ is a $C^{1}$-mapping from $\mathbb{R}^{p+n}$ to $\mathbb{R}^{p}$, with

$$
p=r+|A|+\left|B_{0}(\bar{y})\right| \text {. }
$$

Further, we have $\mathscr{T}(\bar{y}, \bar{\lambda}, \bar{\mu}, \bar{x})=0$, and the Jacobian matrix of $\mathscr{T}$ with respect to $z=(y, \lambda, \mu)$ at the point $(\bar{y}, \bar{\lambda}, \bar{\mu}, \bar{x})$ is nonsingular,

$$
\begin{aligned}
& D_{z} \mathscr{T}(\bar{y}, \bar{\lambda}, \bar{\mu}, \bar{x})=\left[\begin{array}{c:c}
D_{y}^{2} L(\bar{x}, \bar{y}) & E \\
\hdashline E^{\top} & 0
\end{array}\right], \\
& E=-\left[D^{\top} u_{i}(\bar{y}), i \in A \mid D^{\top} v_{j}(\bar{y}), j \in B_{0}(\bar{y})\right] .
\end{aligned}
$$

The latter nonsingularity follows from (ND2) and the fact that $\operatorname{rank}(E)=$ $|A|+\left|B_{0}(\bar{y})\right|$. So, we can apply the implicit function theorem, and we locally obtain a $C^{1}$-mapping $x \mapsto(y(x), \lambda(x), \mu(x))$ such that

$$
\mathscr{T}(y(x), \lambda(x), \mu(x), x) \equiv 0
$$


Moreover, $y(x)$ is a nondegenerate critical point for $G(x, \cdot)_{\mid Y}$. Let us introduce the local marginal function $\phi$ [cf. also Section 1, Eqs. (4)],

$$
\phi(x)=G(x, y(x)) .
$$

Then, it follows that

$$
D \phi(x)=D_{x} G(x, y(x)),
$$

and hence $\phi$ is a $C^{2}$-function.

For later constructions, we also need the structure of the second derivative of $\phi$ at $\bar{x}$ [cf. Ref. 7, Eq. (4.1.12)]:

$$
\begin{aligned}
D^{2} \phi(\bar{x}) & =D_{x}^{2} G(\bar{x}, \bar{y}) \\
& -\left[\begin{array}{c}
D_{x} D_{y}^{\top} G(\bar{x}, \bar{y}) \\
0
\end{array}\right]^{\top} \cdot\left[D_{z} \mathscr{T}(\bar{x}, \bar{y})\right]^{-1} \cdot\left[\begin{array}{c}
D_{x} D_{y}^{\mathrm{T}} G(\bar{x}, \bar{y}) \\
0
\end{array}\right] .
\end{aligned}
$$

Definition 2.6. Suppose that $G \in C^{2}\left(\mathbb{R}^{n} \times \mathbb{R}^{r}, \mathbb{R}\right)$. For $\bar{x} \in M[H, G]$, the extremal set $E_{G}(\bar{x})$ is called nondegenerate if each $\bar{y} \in E_{G}(\bar{x})$ is a nondegenerate minimum for $G(\bar{x}, \cdot)_{\mid Y}$. The reduction principle is said to hold at $\bar{x} \in M[H, G]$ if $E_{G}(\bar{x})$ is nondegenerate.

Suppose that the reduction principle holds at $\bar{x} \in M[H, G]$. Then, each $\bar{y} \in E_{G}(\bar{x})$ is an isolated minimum for $G(\bar{x}, \cdot)_{\mid \gamma}$. Since $Y$ is compact, it follows that $E_{G}(\bar{x})$ is a finite set, say $E_{G}(\bar{x})=\left\{\bar{y}^{1}, \ldots, \bar{y}^{p}\right\}$. Around each point $\left(\bar{x}, \bar{y}^{i}\right)$, the implicit function theorem is applicable; as a consequence, we obtain, in a neighborhood of $\bar{x}$, the representation of the marginal function $\phi_{G}$ as the minimum of the $C^{2}$-functions $\phi_{G}^{i}, i=1, \ldots, p$; cf. Section 1, Eqs. (4).

Unfortunately, the violation of the reduction principle might be stable under perturbations of the problem data. This will now be explained with a simple example arising from an analysis based on singularity theory (cf. Ref. 5).

Example 2.2. Let $Y=[-1,1]$, and let $G \in C^{\infty}\left(\mathbb{R}^{3} \times \mathbb{R}, \mathbb{R}\right)$ be defined by

$$
G(x, y)=y^{4}+x_{1} y^{2}+x_{2} y+x_{3} .
$$

There are no equality constraints, and we are interested in the feasible set $M[G]$ in a neighborhood of the origin. A careful calculation shows that (ELICQ) is satisfied for $x \in M[G]$ near the origin. However, the reduction principle is violated at the point $(\bar{x}, \bar{y})=(0,0)$. The special feature at the origin is represented by means of the mapping $\mathscr{F}$,

$$
\mathscr{F}:(x, y) \mapsto\left(G, D_{y} G, D_{y} D_{y} G, D_{y} D_{y} D_{y} G\right)_{(x, y)} .
$$

Note that $\mathscr{F}$ vanishes at the origin, but the Jacobian matrix $D \mathscr{F}$ at the origin is nonsingular. From this, it follows, basically in virtue of the implicit 
function theorem in Banach spaces (cf. Ref. 13), that the violation of the reduction principle persists under small $C_{s}^{4}$-perturbations of the defining function $G$. For details on the application of the implicit function theorem up to $C_{s}^{4}$-stability, we refer to the exposé in Ref. 11, Chapter 6 .

\section{Lemmas and Proofs of the Theorems}

The proof of the subsequent lemma will be deleted, since it runs almost identically along the lines of the proof of Lemma 2.1 in Ref. 1.

Lemma 3.1. The extended Mangasarian-Fromovitz constraint qualification is invariant under local $C^{1}$-coordinate transformations.

Remark 3.1. Let $\bar{x} \in M[H, G]$, and suppose that (EMFCQ) holds at $\bar{x}$. Then, for a local analysis, we may delete the equality constraints, since the zero set of $H$ is a $C^{1}$-manifold in a neighborhood of $\bar{x}$. In fact, choose $\xi_{j} \in \mathbb{R}^{n}, j=m+1, \ldots, n$, such that the vectors $D^{\top} h_{i}(\bar{x}), i=1, \ldots, m$, and $\xi_{j}$, $j=m+1, \ldots, n$, form a basis for $\mathbb{R}^{n}$. Put

$$
y=\Phi(x)
$$

where

$$
\begin{array}{ll}
y_{i}=h_{i}(x), & i=1, \ldots, m, \\
y_{j}=\xi_{j}^{\top} \cdot(x-\bar{x}), & j=m+1, \ldots, n .
\end{array}
$$

Then, $\Phi$ is of class $C^{1}$ and the Jacobian matrix $D \Phi(\bar{x})$ is nonsingular. Consequently, $\Phi$ is a locally invertible and hence a local $C^{1}$-coordinate transformation sending $\bar{x}$ onto the origin. Now, the set $H^{-1}(0)$ is locally transformed under $\Phi$ to the set $\left\{O_{m}\right\} \times \mathbb{R}^{n-m}$. Note that, if $H$ is of class $C^{k}$, $k \geq 2$, then $\Phi$ is also of class $C^{k}$. Lemma 3.1 implies that (EMFCQ) remains valid in the new coordinates.

The next lemma on the solvability of a compact system of linear inequalities is well known. Let conv ( $)$ denote the convex hull.

Lemma 3.2. See Ref. 14 . Let $K \subset \mathbb{R}^{n}$ be a nonempty compact set. Then, the system of linear inequalities

$$
v^{\top} \xi>0, \quad v \in K,
$$

is solvable if and only if $0 \notin \operatorname{conv}(K)$.

For a locally Lipschitz continuous function $\psi: \mathbb{R}^{n} \rightarrow \mathbb{R}$, let $\partial \psi(x)$ denote the Clarke subdifferential (cf. Ref. 15) evaluated at the point $x$. 
Lemma 3.3. The marginal function $\phi_{G}$ is locally Lipschitz continuous, and for the Clarke subdifferential $\partial \phi_{G}$ the following inclusion holds:

$$
\partial \phi_{G}(x) \subset \operatorname{conv}\left\{D_{x} G(x, y) \mid y \in\left\{w \in Y \mid G(x, w)=\phi_{G}(x)\right\}\right\} .
$$

Proof. The proof follows immediately from Ref. 15, Section 2.8 , using the fact that $G$ is a $C^{1}$-function and that $Y$ is a compact set.

Definition 3.1. Let $h, g: \mathbb{R}^{n} \rightarrow \mathbb{R}$ be given. Then, $h$ and $g$ are said to be Lipschitzian equivalent at $(\bar{x}, \bar{z}) \in \mathbb{R}^{n} \times \mathbb{R}^{n}$ if there exist neighborhoods $\mathcal{U}$ and $\mathscr{V}$ of $\bar{x}$ and $\bar{z}$, respectively, and a bijective mapping $\Phi: \mathscr{U} \rightarrow \mathscr{V}$, both $\Phi$ and $\Phi^{-1}$ Lipschitz continuous, sending $\bar{x}$ onto $z$, such that $h \circ \Phi^{-1}=g$ on $\mathscr{V}$.

The following lemma is a combination of the linearization theorem in Ref. 16, Theorem 2.2 and Ref. 16, Remark 2.2.

Lemma 3.4. Let $h: \mathbb{R}^{n} \rightarrow \mathbb{R}$ be a locally Lipschitz continuous function, and suppose that, at the point $\bar{x} \in \mathbb{R}^{n}$, the origin is not contained in the subdifferential $\partial h(\bar{x})$. Then, $h$ and $g$ are Lipschitzian equivalent at $(\bar{x}, 0)$, where

$$
g\left(y_{1}, \ldots, y_{n}\right)=h(\bar{x})+y_{1} .
$$

In the case where there are no equality constraints $(m=0)$, resp. no inequality constraints $(Y=\varnothing)$, we write $M[G]$, resp. $M[H]$, instead of $M[H, G]$.

Proof of Theorem 2.1. Suppose that (EMFCQ) is satisfied at all points of $M[H, G]$. The proof that $M[H, G]$ is a Lipschitzian manifold (with boundary) consists of a local analysis. Hence, in virtue of Remark 3.1, we can delete the equality constraints. So, we are dealing with a set of the type $M[G]$. Let $\bar{x}$ belong to $M[G]$. If $\phi_{G}(\bar{x})>0$, then by continuity a whole neighborhood of $\bar{x}$ belongs to $M[G]$, and the manifold condition is trivially fulfilled at $\bar{x}$. Now, suppose that $\phi_{G}(\bar{x})=0$. From the validity of (EMFCQ), especially (EMFCQ2), it follows that the system of linear inequalities

$$
D_{x} G(\bar{x}, y) \cdot \zeta>0, \quad y \in E_{G}(\bar{x}),
$$

is solvable. The extremal set $E_{G}(\bar{x})$ is a closed subset of $Y$, and hence is compact. So, it follows from Lemma 3.2 that the origin does not belong to $\operatorname{conv}\left\{D_{x} G(\bar{x}, y) \mid y \in E_{G}(\bar{x})\right\}$. Consequently, from Lemma 3.3 , we see $0 \notin \partial \phi_{G}(\bar{x})$. A combination of Lemma 3.3 and Lemma 3.4 shows that $\phi_{G}$ and $g$ are Lipschitzian equivalent at $(\bar{x}, 0)$, where $g\left(y_{1}, \ldots, y_{n}\right)=y_{1}$. But from this, it follows that the Lipschitzian manifold condition is fulfilled at $\bar{x}$. This completes the proof. 
In order to prove our continuity theorem, we need several preliminary lemmas.

\section{Lemma 3.5.}

(i) The set-valued mapping $\mathscr{E}:(x, G) \mapsto E_{G}(x)$ is upper semicontinuous on $\mathbb{R}^{n} \times C^{1}\left(\mathbb{R}^{n} \times \mathbb{R}^{r}, \mathbb{R}\right)$.

(ii) If (EMFCQ) holds for all $x \in M[H, G]$, then a $C_{s}^{1}$-neighborhood $\mathscr{O} \subset C^{1}\left(\mathbb{R}^{n}, \mathbb{R}^{m}\right) \times C^{1}\left(\mathbb{R}^{n} \times \mathbb{R}^{r}, \mathbb{R}\right)$ exists such that (EMFCQ) is fulfilled for all $x \in M[\tilde{H}, \tilde{G}]$ whenever $(\tilde{H}, \tilde{G}) \in \tilde{O}$.

Proof. Using the compactness of $Y$, this follows directly by a continuity argument. Note that, for the proof of part (ii), we need (i).

Let $\zeta$ be a vector field on an open subset $\mathscr{U}$ of $\mathbb{R}^{n}$, i.e., $\zeta$ is a mapping from $U$ to $\mathbb{R}^{n}$. If $\zeta$ is of the class $C^{k}, k \geq 1$ (resp. locally Lipschitz continuous), then $\zeta$ admits a unique flow (say $\Phi$ ) which is defined on an open neighborhood of $\mathscr{U} \times\{0\} \subset \mathbb{R}^{n} \times \mathbb{R}$, and which is of class $C^{k}$ as well (resp. locally Lipschitz continuous). Recall that, for any $\bar{x} \in \mathcal{U}$, we have

$$
\Phi(\bar{x}, 0)=\bar{x}, \quad \partial / \partial t[\Phi(\bar{x}, t)]=\zeta(\Phi(\bar{x}, t)) ;
$$

this means that $\Phi(\bar{x}, t)$ represents the $\zeta$-trajectory through $\bar{x}$, where $t$ stands for the integration time. For proofs and more details on this subject, see Ref. 17.

In the following lemma, we restrict ourselves to the case where $m=0$ (no equality constraints).

Lemma 3.6. Let $\bar{x} \in M[G]$, such that $\phi_{G}(\bar{x})=0$, be given. Moreover, let $\zeta$ be a $C^{1}$-vector field (with flow $\Phi$ ) on the open neighborhood $U$ of $\bar{x}$. We assume that (EMFCQ) holds at $\bar{x}$, with $\zeta(\bar{x})$ as an EMF-vector. Finally, let $\hat{x} \in \mathcal{U}$ be a point on the $\zeta$-trajectory through $\bar{x}$, i.e., $\hat{x}=\Phi(\bar{x},-\vec{t})$ for some $\bar{t} \in \mathbb{R}$. Then, there exist an open neighborhood $\mathscr{V}$ of $\hat{x}$, a positive real $\alpha$, and a unique Lipschitz continuous function $T: \mathscr{V} \rightarrow[\bar{t}-\alpha, \bar{t}+\alpha]$ satisfying:

(i) $\Phi(x, t)$ is well-defined on $\mathscr{V} \times[\bar{t}-\alpha, \bar{t}+\alpha]$;

(ii) when, for $x \in \mathscr{V}$ arbitrary but fixed, $t$ traverses the interval $[\bar{t}-$ $\alpha, \bar{t}+\alpha]$, the composition $\phi_{G}(\Phi(x, t))$ vanishes iff $t=T(x)$, and moreover it changes sign.

Proof. We only discuss the case where $\bar{x} \neq \hat{x}$ and $\bar{t}>0$; in the other cases, the proof runs along the same lines.

Since $\zeta$ is a $C^{1}$-vector field, its flow $\Phi$ is a $C^{1}$ mapping on a suitably chosen neighborhood of $(\hat{x}, 0)$; this essentially demonstrates (i). Moreover, 
the composition $G(\Phi(x, t), y)$ is of the class $C^{1}$ as well. Since $\zeta(\bar{x})=$ $(\partial / \partial t) \Phi(\hat{x}, \bar{t})$ is an EMF-vector at $\bar{x}$, we have that, for any $\bar{y} \in E_{G}(\bar{x})$,

$$
\begin{aligned}
& G(\Phi(\hat{x}, \bar{t}), \bar{y})=0, \\
& (\partial / \partial t) G(\Phi(\hat{x}, \bar{t}), \bar{y})=D_{x} G(\Phi(\hat{x}, \bar{t}), \bar{y}) \cdot \zeta(\bar{x})>0 .
\end{aligned}
$$

Now, application of the implicit function theorem using the compactness of $E_{G}(\bar{x})$ yields the existence of an open neighborhood $\mathscr{V}$ of $\hat{x}$, a compact neighborhood $\mathscr{W}$ of $E_{G}(\bar{x})$ in $Y$, and a unique $C^{1}$-function $T: \mathscr{V} \times \mathscr{W} \rightarrow$ $[\bar{t}-\alpha, \bar{t}+\alpha]$, with $\alpha>0$, such that

$$
\begin{array}{ll}
T(\hat{x}, y)=\bar{t}, & \text { for all } y \in E_{G}(\bar{x}), \\
G(\Phi(x, t), y)=0, & \text { iff } t=T(x, y), \text { whenever }(x, y) \in \mathscr{V} \times \mathscr{W} .
\end{array}
$$

By a continuity argument (as used for Lemma 3.5), we may assume (possibly after shrinking $\mathscr{V}, \mathscr{W})$ that $E_{G}(x) \subset \mathscr{W}$ and $\zeta(x)$ is an EMF-vector at $x$, whenever $x \in \mathscr{V}$. Now, it is not difficult to prove (using $\bar{t}>0$, and possibly after shrinking $\mathscr{V}, \mathscr{W}, \alpha)$ that, for all $(x, y) \in \mathscr{V} \times \mathscr{W}$, we have

$$
\begin{aligned}
& G(\Phi(x, t), y)<0 \quad(\text { resp. }>0), \\
& \text { if } \bar{t}-\alpha \leq t<T(x, y) \quad \text { (resp. if } T(x, y)<t \leq \bar{t}+\alpha) .
\end{aligned}
$$

We define

$$
T(x)=\min \{T(x, y) \mid y \in \mathscr{W}\} .
$$

Since $T(x, y)$ is of the class $C^{1}$, and thus locally Lipschitzian, we find that $T(x)$ is locally Lipschitz continuous; compare Ref. 15, Section 2.8 . Finally, it is easily shown that $T(x)$ satisfies (ii). This completes the proof.

Proof of Theorem 2.2. As far as the upper semicontinuity of $\mathcal{M}$ is concerned, the proof is a direct consequence of the definition of the $C^{1}$ Whitney topology and the following definitions:

(i) $\quad M[H, G]=\left\{x \in \mathbb{R}^{n} \mid\|H(x)\|=0, \phi_{G}(x) \geq 0\right\}$, where $\|\cdot\|$ stands for Euclidean norm;

(ii) both mappings $(x, H) \mapsto\|H(x)\|$ and $(x, G) \mapsto \phi_{G}(x)$ are continuous w.r.t. the topologies induced by $\|\cdot\|$ and $C_{s}^{1}$.

Next, we prove that $\mathcal{M}$ is lower semicontinuous.

Assume that this is not true. Then, there exists a point $\bar{x} \in M[H, G]$ and a compact neighborhood $U$ of $\bar{x}$ (with smooth boundary) such that, in any open $C_{s}^{1}$-neighborhood $\mathscr{O}$ of $(H, G)$, we can select a pair $\left(H^{0}, G^{0}\right)$ with $M\left(H^{0}, G^{0}\right) \cap \mathscr{U}=\varnothing$. 
In the sequel, we consider only neighborhoods $\mathcal{O}$ such as in Lemma 3.5(ii). This means that, for all $(\tilde{H}, \tilde{G}) \in \mathcal{O}$, we may apply the manifold theorem. We distinguish between two cases.

Case 1. $E_{G}(\bar{x})=\varnothing$. In this case, locally around $\bar{x}$ our feasible sets are merely given by equality constraints. So, we may restrict ourselves to feasible sets of the type $M[\tilde{H}]$, with $\tilde{H}$ in some $C_{s}^{1}$-neighborhood of $H$. Since $H(\tilde{x})=0$ and since (EMFCQ1) holds at $\bar{x}$, we can apply the implicit function theorem for Banach spaces, the actual Banach space being the set of all restrictions $\tilde{H}_{q_{\ell}}$ endowed with the norm $\|\tilde{H}\|_{1}=$ $\max _{\mathcal{U}}\{\|\tilde{H}(x)\|+\|D \tilde{H}(x)\|\}$; cf. also Ref. 7, Theorem 3.1.3. Consequently, there exists an open neighborhood $\tilde{O}$ of $H$ and a $C^{1}$-Frechet differentiable mapping $x(\cdot)$ on $\tilde{\mathcal{O}}$ such that, for all $\tilde{H} \in \tilde{\mathcal{O}}$, we have $x(\tilde{H}) \in M[\tilde{H}] \cap \mathscr{U}$. This latter conclusion, however, is in contradiction with our assumption.

Case 2. $E_{G}(\bar{x}) \neq \varnothing$. In this case, the point $\bar{x}$ is situated on the boundary $\partial M[H, G]$. For $\nu \in \mathbb{N}$ sufficiently large, we treat a special selection $\left\{\mathcal{O}^{\nu}\right\}$ from the above neighborhoods $\mathcal{O}$ of $(H, G)$ such that $(\tilde{H}, \tilde{G}) \in \mathcal{O}^{\nu}$ implies $\|(H-\tilde{H}, G-\bar{G})\|_{1}<1 / \nu$. Here, $\|\cdot\|_{1}$ is defined as above, but now w.r.t. $\mathscr{U} \times \mathscr{B}$, where $\mathscr{B}$ is a compact neighborhood of $Y$ having a smooth boundary. In accordance with our assumption, we choose in each $\mathscr{O}^{\nu}$ a pair $\left(H^{\nu}, G^{\nu}\right)$ such that $\mathcal{M}\left(H^{\nu}, G^{\nu}\right) \cap \mathcal{U}=\varnothing$.

For $\nu$ sufficiently large, we may assume that $H$ and $H^{\nu}$ coincide on $U$. This can be proved with the aid of certain $C^{1}$-diffeomorphisms (constructed from linear homotopies connecting $H^{\nu}$ with $H$ ), which send $M\left[H^{\nu}\right] \cap \mathscr{U}$ onto $M[H] \cap \mathcal{U}$. For these constructions, we merely refer to Ref. 1 or Ref. 11, Chapter 6; here, we use the fact that the mappings $H, H^{\nu}$ are of class $C^{2}$. So, by virtue of Remark 3.1, we may restrict ourselves, locally around $\bar{x}$, to feasible sets of the type $M\left[G^{\nu}\right]$ and $M[G]$.

Now, we proceed as in the proof of Lemma 3.6. Let $\zeta(\bar{x})$ be an EMF-vector at $\bar{x}$ for $G$, and consider the constant vector field $\zeta(x):=\zeta(\bar{x})$. Then, $\zeta$ generates a flow $\Phi$. Interpreting $G$ as an additional variable [and in view of $\bar{x} \in \partial M[G]$, (EMFCQ 2)], we may apply the implicit function theorem for Banach spaces to the equation $G(\Phi(x, t), y)=0$. As in the proof of Lemma 3.6, it follows that, for any $\tilde{G}$ sufficiently close to $G$, the $\zeta$-trajectory through $\bar{x}$ (and restricted to a suitable compact neighborhood, again denoted by $U$, of $\bar{x}$ ) intersects $\partial M[\tilde{G}]$ in exactly one point. In this way, we associate with $\left(G^{\nu}\right)$ a unique sequence of points $\left(x^{\nu}\right)$ such that

$$
x^{\nu} \in \partial M\left[G^{\nu}\right] .
$$

Since $U$ is compact, we may assume $\left(x^{\nu}\right)$ to converge to a point $x^{0} \in \mathcal{U}$. Due to the continuity of the mapping $(x, G) \mapsto \phi_{G}(x)$ and to the very 
selection of the mappings $G^{\nu}$, we arrive at

$$
\phi_{G}\left(x^{0}\right)=\lim _{\nu \rightarrow \infty}\left(\phi_{G^{\nu}}\left(x^{\nu}\right)\right) \text {. }
$$

From (30) and (31), it follows that $x^{0} \in \partial M[G]$. Since the $\zeta$-trajectory through $\bar{x}$ intersects $\partial M[G]$ in exactly one point, it follows that $\bar{x}=x^{0}$. This, however, is violated by our initial assumption that $M\left(G^{\nu}\right) \cap \vartheta=\varnothing$ for all $\nu$.

Finally, in order to prove the last part of the theorem, let $M[H, G]$ be compact, and let $\mathscr{W}$ be a compact neighborhood of $M[H, G]$ in $\mathbb{R}^{n}$. Since $\mathcal{M}$ is upper semicontinuous, $M[\tilde{H}, \tilde{G}]$ is contained in $\mathscr{W}$, and thus is compact, for any $(\tilde{H}, \tilde{G})$ in a suitable $C_{s}^{1}$-open neighborhood $\mathscr{O}$ of $(H, G)$. Now, we are in the position to apply Ref. 9, Chapter 6, Section 6. Both the upper and lower semicontinuity of $\mathscr{M}$ being already demonstrated, we conclude that (after restricting $\tilde{H}$ and $\tilde{G}$ to $W$ and $W \times Y$, respectively) $M$ [as a single-valued mapping from $\mathscr{O}$ to $\left.\mathscr{P}_{c}\left(\mathbb{R}^{n}\right)\right]$ is continuous.

The next lemma is in the same spirit as Ref. 1, Lemma 2.4. The proof (see also Ref. 11, Chapter 7), which is based on Sard's theorem, will be omitted. Let $H \in C^{\infty}\left(\mathbb{R}^{n}, \mathbb{R}^{m}\right)$, and let $\mathscr{C}^{1}, \mathscr{C}^{2}$ be disjoint closed subsets of $\mathbb{R}^{n}$. Let $\tilde{H} \in C^{\infty}\left(\mathbb{R}^{n}, \mathbb{R}^{m}\right)$ belong to $\mathscr{F}\left(\mathscr{C}^{1}, \mathscr{C}^{2}\right)$ if and only if the conditions below are satisfied:

(i) $\tilde{H}$ coincides with $H$ on $\mathscr{C}^{1}$;

(ii) $\operatorname{rank} D \tilde{H}(x)=m$, at all $x \in \mathscr{C}^{2} \cap \tilde{H}^{-1}(0)$.

Lemma 3.7. Let $H \in C^{\infty}\left(\mathbb{R}^{n}, \mathbb{R}^{m}\right)$. Then, for each $k \in \mathbb{N}$, the set $\mathscr{F}\left(\mathscr{C}^{1}, \mathscr{C}^{2}\right)$ intersects every $C_{s}^{k}$-neighborhood of $H$, and $\mathscr{F}\left(\varnothing, \mathscr{C}^{2}\right)$ is $C_{5}^{1}$-open.

\section{Proof of Theorem 2.4 .}

Part (a). We will confine ourselves merely to a sketch of the proof. From Lemma 3.7, we see that there exists a $C_{s}^{\infty}$-open and dense subset $0 \subset C^{\infty}\left(\mathbb{R}^{n}, \mathbb{R}^{m}\right)$ with the following property for $H \in O$ :

$$
\text { rank } D H(x)=m, \quad \text { for all } x \in H^{-1}(0) \text {. }
$$

Hence, for $H \in \mathcal{O}$, the set $H^{-1}(0)$ is a $C^{\infty}$-manifold. As a consequence, we can delete the equality constraints in our further investigation, since the subsequent ideas from transversality theory extend to smooth manifolds.

The set $Y$ need not to be compact in the rest of the proof. Since $Y$ is a smooth manifold with boundary and corners, it suffices to explain the method of proof in the case $Y=\mathrm{H}^{r}$. The main tool consists of application 
of the multijet transversality theorem (cf. Ref. 11, Chapter 7). Let $\bar{x} \in \mathbb{R}^{n}$ be given. Then, for $\bar{y} \in Y=\mathbb{H}^{r}$ to belong to $E_{G}(\bar{x})$, and thus $\bar{y}$ to be a minimum for $G(\bar{x}, \cdot)_{\mid Y}$, the following conditions are necessary:

(i) $\bar{y} \geq 0$,

(ii) $D_{y} G(\bar{x}, \bar{y}) \geq 0$,

(iii) $D_{y} G(\bar{x}, \bar{y}) \cdot \bar{y}=0$.

Formula (32) gives rise to the introduction of the following complementarity subset $E \subset \mathbb{R}^{r} \times \mathbb{R}^{r}$,

$$
\begin{aligned}
E & =\left\{(v, w) \in \mathbb{R}^{r} \times \mathbb{R}^{r} \mid v \geq 0, w \geq 0, v^{\top} w=0\right\} \\
& =\left\{(v, w) \in \mathbb{R}^{r} \times \mathbb{R}^{r} \mid v^{i} \geq 0, w^{i} \geq 0, v^{i} \cdot w^{i}=0, i=1, \ldots, r\right\} .
\end{aligned}
$$

For fixed $i$, the set $\left\{v^{i} \geq 0, w^{i} \geq 0, v^{i} \cdot w^{i}=0\right\}$ can be partitioned (stratified) into three manifolds,

$$
\{(0,0)\},\{0\} \times\left(\mathbb{H}^{1} \backslash\{0\}\right),\left(\mathbb{H}^{1} \backslash\{0\}\right) \times\{0\} .
$$

In this way, the set $E$ has been partitioned into products of smooth manifolds (product stratification), and the highest dimension of such a product manifold is equal to $r$.

Next, consider the 1 -jet extension $j^{1} G$ of $G$,

$$
j^{1} G:(x, y) \mapsto\left(x, y, G, D_{x} G, D_{y} G\right) .
$$

We are interested in those points $(x, y)$ satisfying

$$
G(x, y)=0 \text { and }\left(y, D_{y}^{T} G(x, y)\right) \in E .
$$

These points lie in the subset $\tilde{E}$ of the image space of $j^{1} G$ defined by

$\tilde{E}=\left\{\left(w^{1}, w^{2}, w^{3}, w^{4}, w^{5}\right) \in \mathbb{R}^{n} \times \mathbb{R}^{r} \times \mathbb{R} \times \mathbb{R}^{n} \times \mathbb{R}^{r} \mid w^{3}=0,\left(w^{2}, w^{5}\right) \in E\right\}$.

The set $\tilde{E}$ can again be partitioned into manifolds (in an analogous way as the set $E$ ), and the least number of defining equations (least codimension) is equal to $r+1$ (the entry 1 corresponding to $w^{3}=0$ ). Considering the set $E_{G}(x)$, which consists of $p$ elements, gives rise (roughly speaking) to $p$ copies of mappings $j^{1} G$ as defined in (35). The available dimension then equals $p(n+r)$, corresponding to $p$ copies of $(x, y)$. However, there are at least $(p-1) n+p(r+1)$ restrictions to be fulfilled. The number $(p-1) n$ reflects the fact that the copies $\left(x^{1}, y^{1}\right), \ldots,\left(x^{p}, y^{p}\right)$, corresponding to $E_{Q}(x)$, have to satisfy the $p-1$ systems of equations

$$
x^{1}=x^{2}, \quad x^{2}=x^{3}, \ldots, x^{p-1}=x^{p} .
$$


In the transversal case, which happens to be generic in virtue of the multijet transversality theorem (cf. Ref. 11, Chapter 7), we must have the following inequality:

Number of restrictions (codimension) $\leq$ available dimension.

Hence, in the transversal case the following inequality should be satisfied:

$$
(p-1) n+p(r+1) \leq p(n+r)
$$

and we obtain

$$
p \leq n \text {. }
$$

This shows the generic finiteness of $E_{G}(x)$, and hence (ELICQ1).

The validity of (ELICQ2) follows from a sharpened calculation of restrictions (codimension), taking the $p$ copies of $D_{x} G$ into account. The violation of (ELICQ2), in case $p \leq n$, means that

$$
\operatorname{rank}\left\{D_{x}^{\top} G\left(x, y^{i}\right), i=1, \ldots, p\right\} \leq p-1 .
$$

In the case of the mildest violation, i.e., the case where the least number of restrictions occur in (39), the $n \times p$ matrix in the left-hand side of (39) has rank $p-1$. Now, the set of $n \times p$ matrices, $p \leq n$, having rank equal to $p-1$ constitutes in $\mathbb{R}^{n \cdot p}$ a manifold of codimension $n-p+1$ (cf. Ref. 11). Hence, in the transversal case, the inequality (38) with $n-p+1$ added in the left-hand side should hold, which obviously is false. Therefore, such a situation does not occur generically. This completes the sketch of the proof of Theorem 2.4, Part (a).

Part (b). This follows immediately from Lemma 3.5 , the $C_{s^{-}}^{1}$ density of $C^{\infty}\left(\mathbb{R}^{k}, \mathbb{R}\right)$ in $C^{1}\left(\mathbb{R}^{k}, \mathbb{R}\right)$, and the fact that (ELICQ) implies (EMFCQ).

Remark 3.2. Theorem 2.4 can be refined in the following way. Let us forget about equality constraints at this point. Instead of perturbing the function $G$ on the space $\mathbb{R}^{n} \times \mathbb{R}^{r}$, we can refine the perturbation by firstly deleting, for some $\bar{x} \in \mathbb{R}^{n}$, the set $\{\bar{x}\} \times \mathbb{R}^{r}$ from $\mathbb{R}^{n} \times \mathbb{R}^{r}$. Note that $\mathcal{U}:=$ $\left(\mathbb{R}^{n} \times \mathbb{R}^{r}\right) \backslash\left(\{\bar{x}\} \times \mathbb{R}^{r}\right)$ is an open subset of $\mathbb{R}^{n} \times \mathbb{R}^{r}$. Then, we can endow the space $C^{\infty}(U, \mathbb{R})$ again with the $C_{s}^{\infty}$-topology; and again, the multijet transversality theory can be applied, but now regarding the open subset $U$ as a smooth manifold. Now, let $\mathcal{O}$ be a neighborhood of $G_{\mid q \ell}$ consisting of those elements from $C^{\infty}(\mathcal{U}, \mathbb{R})$ whose functional values and derivatives up to order two differ from $G_{\mid \mathcal{U}}$ up to a continuous positive function $\epsilon(\cdot): \mathcal{U} \rightarrow \mathbb{R}$, where $\epsilon(x, y) \leq d\left((x, y),\{\bar{x}\} \times \mathbb{R}^{r}\right)$ and where $d$ stands for the Euclidean distance. Then, any $\tilde{G} \in \mathcal{O}$ can be extended to a $C^{2}$-function on $\mathbb{R}^{n} \times \mathbb{R}^{r}$, just by defining the derivatives up to order two on the set $\{\bar{x}\} \times \mathbb{R}^{r}$ to be 
those of $G$. This allows quite delicate perturbations which will be used in the proof of Theorem 2.3.

For the next lemma, see Ref. 1, Lemma 2.3.

Lemma 3.8. Let $f \in C^{1}\left(\mathbb{R}^{n}, \mathbb{R}\right)$, and let $\bar{x} \in \mathbb{R}^{n}$. Then, every $C_{s}^{1}$-neighborhood of $f$ contains an $\tilde{f}$ satisfying:

(i) $\tilde{f} \in C^{\infty}\left(\mathbb{R}^{n}, \mathbb{R}\right)$;

(ii) $\tilde{f}(\bar{x})=f(\bar{x}), \quad D \tilde{f}(\bar{x})=D f(\bar{x})$.

Remark 3.3. In the proof of Theorem 2.3, we sometimes use the expression "we add locally at $K$ a function $g$ to the function $h$," where $K$ is a compact set. By this expression, we mean that we actually add the function $g(x) \xi(x)$ to $h(x)$, where $\xi(x)$ is a $C^{\infty}$-function having the following properties:

(i) $0 \leq \xi(x) \leq 1$;

(ii) $\xi$ has a compact support;

(iii) $\xi(x)$ is identically equal to one in some neighborhood of $K$.

For the proof of our stability theorem, we need one more lemma.

Lemma 3.9. Let $\bar{x} \in M[G]$. Moreover, we suppose that $E_{G}(\bar{x})$ is nonempty and finite, say $E_{G}(\bar{x})=\left\{\bar{y}^{1}, \ldots, \bar{y}^{p}\right\}$. Then, any open $C_{s}^{1}$-neighborhood $O$ of $G$ contains an element $\tilde{G}$ such that the following properties hold:

(i) $\quad \tilde{G} \in C^{\infty}\left(\mathbb{R}^{n} \times \mathbb{R}^{r}, \mathbb{R}\right)$;

(ii) $\bar{x} \in M[\tilde{G}]$, and $E_{\tilde{G}}(\bar{x})=\left\{\bar{y}^{1}, \ldots, \bar{y}^{p}\right\}$;

(iii) each $\bar{y}^{i} \in E_{\tilde{G}}(\bar{x})$ is a nondegenerate minimum for $\bar{G}(\bar{x}, \cdot)_{\mid Y}$;

(iv) $D_{x} \tilde{G}\left(\bar{x}, \bar{y}^{i}\right)=D_{x} G\left(\bar{x}, \bar{y}^{i}\right), i=1, \ldots, p$.

Proof. The proof consists of three steps, each providing us with an arbitrarily good approximation of $G$.

Step 1. In this step, we proceed as in Remark 3.2. Let the open subset $\mathcal{U}$ of $\mathbb{R}^{n} \times \mathbb{R}^{r}$ be defined by

$$
\mathcal{U}=\left(\mathbb{R}^{n} \times \mathbb{R}^{r}\right) \backslash\left\{\left(\bar{x}, \bar{y}^{i}\right) \mid i=1, \therefore, p\right\},
$$

and consider a positive function $\epsilon: \mathscr{U} \rightarrow \mathbb{R}$ with the property that

$$
\begin{array}{r}
\epsilon(x, y) \leq \min \left\{\left\|(x, y)-\left(\bar{x}, \bar{y}^{i}\right)\right\|, i=1, \ldots, p, G(\bar{x}, y)\right\}, \\
\text { whenever }(x, y) \in \mathcal{U} \text { admits } y \in Y .
\end{array}
$$


The $C_{s}^{1}$-density of $C^{\infty}(\mathcal{U}, \mathbb{R})$ in $C^{1}(U, \mathbb{R})$ yields the existence of a function $\hat{G} \in C^{\infty}(\mathscr{U}, \mathbb{R})$ such that $G-\hat{G}$ is controlled, in the sense of the $C_{s}^{1}$-topology, by $\epsilon(\cdot)$. We extend $\hat{G}$ to the whole $\mathbb{R}^{n} \times \mathbb{R}^{r}$ by defining

$$
\hat{G}\left(\bar{x}, \bar{y}^{i}\right)=0=G\left(\bar{x}, \bar{y}^{i}\right), \quad i=1, \ldots, p .
$$

A straightforward verification shows that the extended function (again denoted $\hat{G})$ is continuously differentiable at $\left(\bar{x}, \bar{y}^{i}\right)$, and moreover,

$$
\begin{aligned}
& \hat{G}(\bar{x}, y)>0, \quad \text { for all } y \in Y \backslash E_{G}(\bar{x}), \\
& D_{x} \hat{G}\left(\bar{x}, \bar{y}^{i}\right)=D_{x} G\left(\bar{x}, \bar{y}^{i}\right), \\
& \bar{x} \in M[\hat{G}] .
\end{aligned}
$$

So, we are able to approximate our function $G$ arbitrarily well (in the $C_{s}^{1}$-sense) by a function $\hat{G}$ which already is of class $C^{\infty}$ on $\mathcal{U}$ [and still fulfills (ii) and (iv)].

Step 2. In this step, we approximate $\hat{G}$ (see Step 1 ) by $\bar{G}$, which is $C^{\infty}$ on the whole $\mathbb{R}^{n} \times \mathbb{R}^{r}$. The function $\bar{G}$ differs from $\hat{G}$ only in a neighborhood of the points $\left(\bar{x}, \bar{y}^{i}\right), i=1, \ldots, p$. Moreover,

$$
D_{x} \bar{G}\left(\bar{x}, \bar{y}^{i}\right)=D_{x} G\left(\bar{x}, \bar{y}^{i}\right), \quad i=1, \ldots, p .
$$

By $B_{i}(3 \delta)$, we denote open balls in $\mathbb{R}^{n} \times \mathbb{R}^{r}$, centered at $\left(\bar{x}, \bar{y}^{i}\right)$ with radii $3 \delta, \delta$ being chosen so small that the closures $\bar{B}_{i}(3 \delta)$ are disjoint.

From the special features of $Y$ (cf. Section 2), it follows that, locally around each $\bar{y}^{i}$ and w.r.t. suitable $C^{\infty}$-coordinates, the set $Y$ can be regarded as an open $\mathbb{H}^{l} \times \mathbb{R}^{r-l}$-neighborhood of the origin $0\left(\bar{y}^{i}\right.$ corresponding to 0 , and $l$ denoting the number of inequality constraints for $Y$ which are active at $\vec{y}^{i}$ ); see also Remark 3.1 and Ref. 7, Lemma 3.1.2.

Possibly after shrinking $\delta$, we may write $y=(\hat{y}, \tilde{y}) \in \mathbb{H}^{\prime} \times \mathbb{R}^{r-t}$, whenever $(x, y) \in \vec{B}_{i}(3 \delta)$ and $y \in Y$, especially $\bar{y}^{i}=(0,0)$. On $B_{i}(\delta)$, we define the function $R_{i}$ as follows:

$$
R_{i}(x, y)=-\hat{G}(x, y)+D_{x} \hat{G}(\bar{x}, 0) \cdot(x-\bar{x})+D_{\hat{y}} \hat{G}(\tilde{x}, 0) \cdot \hat{y} .
$$

Note that $R_{i}$ is of the class $C^{1}$ at $(\bar{x}, 0)$, whereas $R_{i}$ is of class $C^{\infty}$ (smooth) everywhere else on $B_{i}(\delta)$.

For $\epsilon>0$ sufficiently small, we may assume that the lower level set $\boldsymbol{U}_{\epsilon}$, given by $\hat{G}(x, y) \leq \epsilon$, is contained in $B_{i}(\delta)$. Then, using a partition-of-theunity argument (Ref. 7), we extend $R_{i} \mid u_{\epsilon}$ smoothly to a function (again denoted $R_{i}$ ) on $B_{i}(3 \delta)$ which outside $U_{\epsilon}$ is controlled (in the $C_{s}^{1}$-sense) by $\epsilon$ and the $C^{1}$-norm on $B_{i}(\delta)$ of the original $R_{i}$; moreover, $R_{i}$ vanishes on the ring $B_{i}(3 \delta) \backslash \bar{B}_{i}(2 \delta)$. Now, we extend $R_{i}$ to a global function by defining $R_{i}:=0$ outside $B_{i}(3 \delta)$. 
Put $\bar{G}_{i}=\hat{G}+R_{i}$. Then, $\bar{G}_{i}$ is of the class $C^{\infty}$ on the whole $B_{i}(3 \delta)$. Moreover, by shrinking $\delta$ and $\epsilon$ appropriately, we assure $\bar{G}_{i}$ to be arbitrarily close (in the $C_{s}^{1}$-sense) to $\hat{G}$.

Now, it is easily seen that, for $i=1, \ldots, p$,

$$
\begin{array}{ll}
D_{x} \bar{G}_{i}=D_{x} \hat{G}, & \text { at }(\bar{x}, 0)=\left(\bar{x}, \bar{y}^{i}\right), \\
\bar{G}_{i}(\bar{x}, y) \geq 0, & \text { for all } y \in Y, \\
\bar{G}_{i}(\bar{x}, y)=0, & \text { if } y=\bar{y}^{i}, i=1, \ldots, p .
\end{array}
$$

Finally, we add all functions $R_{i}$ to $\hat{G}$ and obtain the announced function $\bar{G}$.

Step 3. In this final step, we approximate $\bar{G}$ (see Step 2 ) by a function $\tilde{G}$ as required in the lemma.

We deal with the linear $Y$-coordinates around $\bar{y}^{i}$ as introduced in the preceding step, and emphasize that, w.r.t. these $Y$-coordinates, the Hessian matrix of $\tilde{G}$ is completely annihilated in some neighborhood of $(\bar{x}, 0)=$ $\left(\bar{x}, \tilde{y}^{i}\right)$. By $\hat{y}^{\prime}$, we denote the partition of $\hat{y}$ corresponding to the active inequality constraints at $\bar{y}^{i}$ with vanishing Lagrange multipliers for $G(\bar{x}, \cdot)$; cf. Definition 2.5.

Firstly, we perturb $\bar{G}$ locally around each $\left(\bar{x}, \bar{y}^{i}\right)$ in such a way that the resulting function $\overline{\bar{G}}$ fulfills condition (ND1) of Definition 2.5 [without losing the already obtained qualifications (i), (ii), and (iv)]. This is done by adding to $\bar{G}$, locally at each $\bar{B}_{i}(3 \delta)$, a linear function of the type

$$
\left(\hat{y}^{\prime}\right)^{\top} \cdot \eta^{\prime}
$$

where the positive entries of the vector $\eta^{\prime}$ are chosen suitably small. Finally, in order to assure condition (ND2) of Definition 2.5 , we add to $\overline{\bar{G}}$, locally at each $\bar{B}_{i}(3 \delta)$, a quadratic polynomial of the type

$$
(\tilde{y})^{\top} \cdot A \cdot \tilde{y}
$$

where the matrix $A$ is symmetric, positive-definite with sufficiently small entries. In this way, we obtain our function $\tilde{G}$ as required.

\section{Proof of Theorem 2.3.}

Sufficiency. The proof is essentially based on Theorem 2.1, Lemmas 3.5 , and 3.6, these results being direct generalizations, to the case where $Y$ is an infinite compact index set, of results which were already obtained in Ref. 1. For the rest, the proof is identical to that in Ref. 1. Therefore, the proof of the sufficiency part will be deleted here.

Necessity. Suppose that (EMFCQ) is violated at $\bar{x} \in M[H, G]$. The main idea is to approximate $H, G$ (arbitrary well in the $C_{s}^{1}$-sense) by means 
of mappings $\tilde{H}, \tilde{G}$, (resp. $\hat{H}, \hat{G})$, such that $M[\tilde{H}, \tilde{G}]$ is not homeomorphic with $M[\hat{H}, \hat{G}]$. In the sequel, we assume (no loss of generality) that $\bar{x}=0$.

Case 1. Rank $D H(\bar{x})<m$. If $E_{G}(\bar{x}) \neq \varnothing$, we add locally at $K:=\{\bar{x}\} \times Y$ a small positive constant to the function $G$. Then, we approximate $G, H$ by means of $C^{\infty}$-function $\tilde{G}, \tilde{H}$ such that

$$
\tilde{H}(\bar{x})=H(\bar{x}) \text { and } D \tilde{H}(\bar{x})=D H(\bar{x}) ;
$$

cf. Lemma 3.8; we denote the function $\tilde{G}, \tilde{H}$ again by $G, H$. The situation now is the following: $M[H, G]$ is compact, the functions $H, G$ are of class $C^{\infty}, \tilde{x} \in M[H, G]$, and $E_{G}(\bar{x})=\varnothing$. Next, as in Ref. 1 , we add locally at $\bar{x}=0$ linear functions (with arbitrarily small derivatives) to the functions $h_{i}, i=1, \ldots, m$, such that, after this perturbation, we have at $\bar{x}$ :

$$
\operatorname{rank}(D H)=\operatorname{rank}\left(D^{\top} h_{1}|\cdots| D^{\top} h_{m-1}\right)=m-1 .
$$

Then, in a neighborhood of $\bar{x}$, the common zero set of the functions $h_{1}, \ldots, h_{m-1}$, denoted by $M[m-1]$, is a smooth manifold (cf. also Remark 3.1 ); moreover, the point $\bar{x}$ is a critical point for $h_{m[M[m-1]}$ (cf. Definition 2.5). Then, we add locally at $\bar{x}=0$ a homogeneous polynomial of degree two (with arbitrarily small coefficients) to the function $h_{m}$ in order that, after this perturbation, the point $\bar{x}$ is a nondegenerate critical point for $h_{\left.m_{\mid M[m-1}\right]}$; cf. Definition 2.5 ; only (ND2) is now relevant. Finally, we approximate $G$ and, outside a sufficiently small neighborhood of $\bar{x}$, we approximate $H$, both in the $C_{s}^{1}$-sense, by means of $\tilde{H}, \tilde{G}$, such that $M[\tilde{H}, \tilde{G}]$ is compact, $E_{\tilde{G}}(\bar{x})=\varnothing$ (cf. Lemma 3.7); moreover (cf. Theorem 2.4), (ELICQ) should be satisfied at all points of $M[\tilde{H}, \tilde{G}] \backslash\{\bar{x}\}$. From the very construction, we see that the feasible set $M[\tilde{H}, \tilde{G}]$ coincides with $M[H]$ in some neighborhood of $\bar{x}$. The following reasoning is analogous to that in Ref. 1, but for better understanding we have to repeat it here. We distinguish between two subcases.

Case 1a. The point $\bar{x}$ is a local minimum (resp. local maximum) for $\tilde{h}_{m_{\mid M[m-1]}}$; recall that $h_{m}$ and $\tilde{h}_{m}$ coincide in a neighborhood of $\bar{x}$. Since $\bar{x}$, as a nondegenerate critical point, is isolated, we see that $\bar{x}$ is an isolated feasible point. If we add locally at $\bar{x}$ a small negative (resp. positive) constant to the function $\tilde{h}_{m}$, thereby obtaining the function $\hat{h}_{m}$, we get: $M[\tilde{H}, \tilde{G}]=$ $M[\hat{H}, \hat{G}] \cup\{\bar{x}\}$ (disjoint union), where $\hat{h}_{i}=\tilde{h_{i}}, i \neq m$, and $\hat{G}=\tilde{G}$. But then the number of connected components of $M[\hat{H}, \hat{G}]$ [finite, since $M[\hat{H}, \hat{G}]$ is a compact Lipschitzian manifold with boundary] is one less than the corresponding number for $M[\tilde{H}, \tilde{G}]$. Consequently, $M[\tilde{H}, \tilde{G}]$ and $M[\hat{H}, \hat{G}]$ cannot be homeomorphic.

Case 1b. The point $\bar{x}$ is neither a local minimum nor a local maximum for $\tilde{h}_{m_{\mid M[m-1]}}$. Then, application of the Morse lemma, in local coordinates 
for $M[m-1]$ (cf. Ref. 7 ), yields that the feasible set $M[\tilde{H}, \tilde{G}]$ in a neighborhood of $\bar{x}$ is represented by means of the following set $\sum$ in $\mathbb{R}^{n-m+1}$ :

$$
\Sigma=\left\{w \in \mathbb{R}^{n-m+1} \mid \sum_{i=1}^{n-m+1} \pm w_{i}^{2}=0\right\},
$$

where in (50) at least one positive as well as one negative square appears. But then, using homotopy groups, it is shown in Ref. 1, Lemma 2.10 that $\sum$ is not the germ of a topological manifold at $w=0$. Next, we add an arbitrarily small constant to $\tilde{h}_{m}$, locally at $\bar{x}$, and we obtain, in view of $(50)$, that (ELICQ) is satisfied at all points of $M[\hat{H}, \hat{G}]$; hence, in virtue of Theorem $2.1, M[\hat{H}, \hat{G}]$ is actually a Lipschitzian manifold with boundary. Here, $\hat{h}_{i}=\tilde{h}_{i}, i \neq m, \hat{h}_{m}$ is the perturbed $\tilde{h}_{m}$, and $\hat{G}=\tilde{G}$. Consequently, we conclude that $M[\hat{H}, \hat{G}]$ is not homeomorphic with $M[\tilde{H}, \tilde{G}]$.

Case 2. Rank $D H(\bar{x})=m$. In this case, the set $H^{-1}(0)$ is (eventually after smooth approximation, cf. Lemma 3.8) a $C^{\infty}$-manifold in a neighborhood of $\bar{x}$ and so, for a further local analysis, we may delete $H$, i.e., we put $m=0$.

Since (EMFCQ), in particular (EMFCQ2), is not satisfied at $\bar{x}$, it follows that $E_{G}(\bar{x}) \neq \varnothing$ and that the system of linear inequalities

$$
D_{x} G(\bar{x}, y) \cdot \xi>0, \quad y \in E_{G}(\bar{x})
$$

is not solvable. Since $E_{G}(\tilde{x})$ is compact, we can apply Lemma 3.2, and hence,

$$
0 \in \operatorname{conv}\left\{D_{x} G(\bar{x}, y) \mid y \in E_{G}(\bar{x})\right\} \text {. }
$$

Next, we choose a minimal (finite) subset, say

$$
\left\{\bar{y}^{1}, \ldots, \bar{y}^{p}\right\} \subset E_{G}(\bar{x}), \quad \text { s. t. } 0 \in \operatorname{conv}\left\{D_{x} G\left(\bar{x}, \bar{y}^{i}\right), i=1, \ldots, p\right\} .
$$

Consequently, there exist unique numbers $\bar{\alpha}^{i} \in \mathbb{R}, i=1, \ldots, p$, such that

$$
\sum_{i=1}^{p} \bar{\alpha}^{i} D_{x} G\left(\bar{x}, \bar{y}^{i}\right)=0, \quad \bar{\alpha}^{i}>0, \quad \sum_{i=1}^{p} \bar{\alpha}^{i}=1 .
$$

Put $\mathscr{A}=\left\{\left(\bar{x}, \bar{y}^{1}\right), \ldots,\left(\bar{x}, \bar{y}^{p}\right)\right\}$, and let $\mathscr{V}$ be a bounded, open neighborhood of $\{\bar{x}\} \times Y$. Then, by a classical result due to Whitney (cf. Ref. 18), there exists a function $\psi \in C^{\infty}\left(\mathbb{R}^{n} \times \mathbb{R}^{r}, \mathbb{R}\right)$ such that

$$
\begin{aligned}
& \psi(x, y) \geq 0, \\
& \psi(x, y)=0, \quad \text { iff }(x, y) \in \mathscr{A} \cup\left[\left(\mathbb{R}^{n} \times \mathbb{R}^{r}\right) \backslash \mathscr{V}\right] .
\end{aligned}
$$

Note that (53) implies that

$$
D \psi\left(\bar{x}, \bar{y}^{i}\right)=0, \quad i=1, \ldots, p
$$


If the positive real $\lambda$ is chosen suitably small, then the function $\bar{G}:=G+\lambda \psi$ will be arbitrary $C_{s}^{1}$-close to $G$. Moreover, we obviously have

$$
\begin{aligned}
& \bar{x} \in M[\bar{G}], \quad E_{\bar{G}}(\bar{x})=\left\{\bar{y}^{1}, \ldots, \bar{y}^{p}\right\}, \\
& D_{x} \bar{G}\left(\bar{x}, \bar{y}^{i}\right)=D_{x} G\left(\bar{x}, \bar{y}^{i}\right), \quad i=1, \ldots, p .
\end{aligned}
$$

Now, we may apply Lemma 3.9 to the $C^{1}$-function $\bar{G}$. We approximate $\bar{G}$ arbitrarily well by a $C^{\infty}$-function (again denoted $G$ ), such that $G$ fulfills (54), (55), thus also (52), and moreover,

$$
\text { each } \bar{y}^{i} \text { is nondegenerate as a local minimum for } G(\bar{x}, \cdot)_{\mid Y} \text {. }
$$

Let $\phi^{i}(x):=G\left(x, y^{i}(x)\right)$ denote the local marginal functions as introduced in Section 1, Eqs. (4). Then, in a neighborhood $U$ of $\bar{x}$, the feasible set $M[H, G]$ is equal to the following set:

$$
\left\{x \mid \max _{i} f^{i}(x) \leq 0, i=1, \ldots, p\right\}, \quad f^{i}(x):=-\phi^{i}(x) .
$$

Recalling (20) and (21), we have

$$
D f^{i}(\bar{x})=-D_{x} G\left(\bar{x}, \bar{y}^{i}\right), \quad D^{2} f^{i}(\bar{x})=-D_{x}^{2} G\left(\bar{x}, \bar{y}^{i}\right)+W^{i},
$$

where the entries of the matrix $W^{i}$ consist of derivatives with respect to $y$ and mixed derivatives with respect to $x$ and $y$. We proceed by adding to the function $G$, locally at $K(=\{\bar{x}\} \times Y)$, a function of the type $(1 / 2)(x-$ $\bar{x})^{\top} \cdot C \cdot(x-\bar{x}), C$ being a symmetric $n \times n$ matrix with arbitrarily small entries. The matrix $C$ is chosen in such a way that the matrix

$$
V^{\top} \cdot\left(\sum_{i=1}^{n} \bar{\alpha}^{i}\left(D^{2} f^{i}(\bar{x})+C\right)\right) \cdot V
$$

becomes nonsingular, $V$ being a fixed chosen matrix whose columns form a basis for the linear space

$$
\bigcap_{i=1}^{p} \operatorname{Ker} D f^{i}(\bar{x})=\bigcap_{i=1}^{p} \operatorname{Ker} D_{x} G\left(\bar{x}, \bar{y}^{i}\right) .
$$

After the choice of the matrix $C$, let $k$ denote the number of negative eigenvalues of the nonsingular matrix in (59).

Following Remark 3.2, we perturb $H$ outside $\{\tilde{x}\}$ and the $C^{\infty}{ }^{-}$function $G$ outside the set $\{\bar{x}\} \times \mathbb{R}^{r}$ such that:

(i) the perturbed function $\tilde{G}$ is a $C^{2}$-function on $\mathbb{R}^{n} \times \mathbb{R}^{r}$;

(ii) the derivatives up to order two of $G$ and $\tilde{G}$ coincide on $\{\bar{x}\} \times \mathbb{R}^{r}$;

(iii) $M[\tilde{H}, \tilde{G}]$ is compact;

(iv) (ELICQ) holds at all points of $M[\tilde{H}, \tilde{G}] \backslash\{\bar{x}\}$. 
Denote $\tilde{H}, \tilde{G}$ again by $H, G$. Recall that the implicitly perturbed local marginal functions are also of class $C^{2}$ (cf. Section 2). Now, we are in the following situation: in a neighborhood of $\bar{x}$, the feasible set has the structure of a lower level set of a function of maximum type in a neighborhood of a nondegenerate $(+)$ Kuhn-Tucker point of quadratic index $k$ (cf. Ref. 7, Chapter 4). From this point, we can complete the proof as in Ref. 1. The local addition of an arbitrarily small positive (resp. negative) constant to the function $G$ at $K$ as above gives rise to two feasible sets, $M[\tilde{H}, \tilde{G}]$ and $M[\hat{H}, \hat{G}]$, respectively, both of them compact and satisfying (ELICQ) at all feasible points with the property that $M[\tilde{H}, \tilde{G}]$ is homotopy-equivalent to $M[\hat{H}, \hat{G}]$ with a $k$-cell attached. But then (cf. Ref. 7 ), $M[\tilde{H}, \tilde{G}]$ and $M[\hat{H}, \hat{G}]$ do not have the same homotopy type as compact Lipschitzian manifolds with boundary. As a consequence, they cannot be homeomorphic. This completes the proof.

\section{References}

1. Guddat, J., Jongen, H. T., and RueCkMANN, J., On Stability and Stationary Points in Nonlinear Optimization, Journal of the Australian Mathematical Society, Series B, Vol. 28, pp. 36-56, 1986.

2. Mangasarian, O. L., and Fromovitz, S., The Fritz John Necessary Optimality Conditions in the Presence of Equality and Inequality Constraints, Journal of Mathematical Analysis and Applications, Vol. 17, pp. 37-47, 1967.

3. WeTtERLING, W. W. E., Definitheitsbedingungen für relative Extrema bei Optimierungs-und Approximationsaufgaben, Numerische Mathematik, Vol. 15, pp. 122-136, 1970.

4. HeTtICH, R., and ZeNCKE, P., Numerische Methoden der Approximation und semi-infiniten Optimierung, Teubner Taschenbücher, Stuttgart, Germany, 1982.

5. Jongen, H. T., and ZWIER, G., On the Local Structure of the Feasible Set in Semi-Infinite Optimization, Parametric Optimization and Approximation, Edited by B. Brosowski and F. Deutsch, Birkhäuser Verlag, Basel, Switzerland, pp. 185$202,1985$.

6. ZWIER, G., Structural Analysis in Semi-Infinite Programming, Dissertation, University of Twente, Twente, Holland, 1987.

7. JongEN, H. T., JONKER, P., and TWILT, F., Nonlinear Optimization in $\mathbb{R}^{n}$, Vol. 1: Morse Theory, Chebychev Approximation, Peter Lang Verlag, Frankfurt am Main, Germany, 1983.

8. Hirsch, M. W., Differential Topology, Springer-Verlag, New York, New York, 1976.

9. BERGE, C., Espaces Topologique, Functions Multivoques, Dunod, Paris, France, 1966.

10. Bank, B., Guddat, J., Klatte, D., Kummer, B., and TAmmer, K., Nonlinear Parametric Optimization, Akademie-Verlag, Berlin, Germany, 1982. 
11. Jongen, H. T., Jonker, P., and Twilt, F., Nonlinear Optimization in $\mathbb{R}^{n}$, Vol. 2: Transversality, Flows, Parametric Aspects, Peter Lang Verlag, Frankfurt am Main, Germany, 1986.

12. FIACCO, A. V., Introduction to Sensitivity and Stability Analysis in Nonlinear Programming, Academic Press, New York, New York, 1983.

13. LJUSTERNIK, L. A., and Sobolew, W. I., Elemente der Funktionalanalysis, Akademie-Verlag, Berlin, Germany, 1960.

14. Cheney, E. W., Introduction to Approximation Theory, McGraw-Hill, New York, New York, 1966.

15. Clarke, F. H., Optimization and Nonsmooth Analysis, John Wiley and Sons, New York, New York, 1983.

16. JoNGEN, H. T., and PAllaschKe, D., On Linearization and Continuous Selections of Functions, Optimization, Vol. 19, pp. 343-353, 1988.

17. AmAnN, H., Gewöhnliche Differentialgleichungen, Walter de Gruyter, Berlin, Germany, 1983.

18. Broecker, T., and LANDER, L., Differentiable Germs and Catastrophes, Cambridge University Press, Cambridge, England, 1975. 